\title{
PROPUESTA TECNOLÓGICA PARA LA IMPLEMENTACIÓN DE UNA PLANTA FOTOVOLTAICA DE 4.5 kWP PARA LA COMUNIDAD RURAL ALTOANDINA SAN FRANCISCO DE RAYMINA, AYACUCHO-PERÚ
}

\author{
TECHNOLOGICAL PROPOSAL FOR THE IMPLEMENTATION OF A \\ 4.5 kW PHOTOVOLTAIC PLANT FOR THE RURAL COMMUNITY \\ ALTOANDINA SAN FRANCISCO DE RAYMINA, AYACUCHO-PERU
}

\author{
Carlos Salvador-Tixe ${ }^{11}$ (i), Manfred Horn-Mutschler ${ }^{1}$ (I) \\ ${ }^{1}$ Facultad de Ciencias, Universidad Nacional de Ingeniería, Lima, Perú
}

Recibido (Received): 23/02/ 2021 Aceptado (Accepted): 18/06/2021

\section{RESUMEN}

El presente documento forma parte de un trabajo de investigación, en donde se estudia la situación energética de la comunidad rural altoandina San Francisco de Raymina, ubicada en la Región Ayacucho a 3798 msnm. Se describe y analiza la situación actual del suministro de energía eléctrica de la localidad. Sobre la base de un diagnóstico situacional, identificando las necesidades (uso y consumo) de energía eléctrica de la población, y una evaluación del potencial energético renovable del lugar, se plantea una solución tecnológica limpia y viable. Particularmente, como la comunidad tiene un suministro de electricidad muy limitado, típico de muchos pueblos similares, insuficiente para el desarrollo de actividades productivas, se propone una instalación fotovoltaica local, a fin de reemplazar el sistema de red convencional y satisfacer la demanda local de manera sustentable.

Palabras clave: Planta fotovoltaica, Energía solar, Tecnología fotovoltaica, Sistema fotovoltaico conectado a la red.

\section{ABSTRACT}

This document is part of a research work, where the energetic situation of the rural community San Francisco de Raymina, located in the Ayacucho Region at 3798 masl, is evaluated. The current situation of electric energy supply in the locality is described and analyzed. Based on a situational diagnosis, identifying the needs (use and consumption) of electric energy of the population, and an evaluation of the renewable energy potential of the place, a clean and viable technological solution is proposed. Particularly, as the community has very limited electricity supply, typical for many similar villages, insufficient for the development of productive activities, a local photovoltaic installation is proposed, in order to replace the conventional grid system and to satisfy the local demand in a sustainable way.

Keywords: Photovoltaic plant, Solar energy, Photovoltaic technology, Grid-connected photovoltaic system.

\section{INTRODUCCIÓN}

En Perú, la población más pobre y vulnerable se ubica en las zonas altoandinas, donde predominan comunidades cuya población vive bajo condición de pobreza extrema. Según cifras del INEI, en referencia a la población peruana que habita en condición de pobreza y pobreza extrema, a la fecha alrededor de 966 mil no cuentan con energía eléctrica (3.0\% total de peruanos), 487 mil utilizan velas para alumbrarse $(1.5 \%$ total de peruanos), alrededor de 1.6 millones utilizan leña para la cocción de alimentos, calentamiento de agua y calefacción de sus hogares $(5.0 \%$ total de peruanos). 660 mil pobladores del Perú, que habitan en las zonas altoandinas, viven bajo condiciones de pobreza extrema, caracterizado, entre otros, por la falta de acceso a la energía [1]. Algunas poblaciones, de estos sectores, cercanas a los puntos de alimentaciones de red ya cuentan con energía eléctrica; sin embargo, esto no es suficiente para hacer frente a sus necesidades productivas, tal como es el caso de la comunidad rural en estudio. La problemática de la población en condición de pobreza extrema se manifiesta fundamentalmente en la falta de energía. El suministro de servicios energéticos básicos adecuados es un requisito indispensable para facilitar el proceso de 
mejora y el progreso de los sectores más pobres y vulnerables del Perú.

En la última década, el estado peruano, instituciones privadas y organismos no gubernamentales (ONGs), han llevado a cabo diversos proyectos para la energización de zonas rurales empleando como fuente energética a las energías renovables. En la actualidad, gran parte de los proyectos desarrollados colapsaron o se encuentran en un estado precario. Algunas de las razones por las que no tuvieron éxito fueron: por desconocimiento del sector y por no contar con un diagnóstico energético adecuado de la zona. Se tiende a replicar soluciones estandarizadas que, difícilmente, se adaptan a las necesidades de cada zona. Distintas experiencias alrededor del mundo, demuestran que proyectos no adecuados pueden ser más perjudiciales que beneficiosos [2], [3]. Analizar el tema energético desde la óptica territorial presenta múltiples ventajas ya que permite, entre otras cosas: Identificar los recursos energéticos renovables potenciales, analizar la demanda energética e identificar las prioridades energéticas [4].

La falta de energía dentro del territorio rural incide de manera negativa en las condiciones de calidad de vida de los pobladores, dificulta el desarrollo de las actividades domésticas y la productividad agropecuaria [5]. La energía desempeña una importante función para el desarrollo económico de las comunidades rurales. El consumo de energía es uno de los grandes medidores del progreso y bienestar de una sociedad [6]. Por otra parte, el territorio peruano es privilegiado por contar con un alto potencial energético renovable aprovechable [7]. Las regiones altoandinas, cuentan con un gran potencial de energía solar, mayor de $5.0 \mathrm{kWh} \cdot \mathrm{m}^{-2} \cdot \mathrm{día}^{-1}$, en promedio anual, durante todo el año [8]. El nivel de irradiancia diaria por año se registra en $221 \mathrm{~W} \cdot \mathrm{m}^{-2}$. Se estima un potencial aprovechable no menor a $25000 \mathrm{MW}$ [9].

La aplicación de tecnologías renovables es una gran opción para la energización de zonas rurales altoandinas, donde el potencial energético renovable se presenta como una fuente energética aprovechable. El uso de estas tecnologías puede mejorar y proveer un servicio eficiente, confiable y de calidad para mejorar las condiciones de los servicios básicos energéticos del sector.

Por todo lo anteriormente expuesto, el presente trabajo confronta el problema de déficit de energía eléctrica que presenta la comunidad en estudio y propone una solución tecnológica viable, que aprovecha el potencial de la energía solar del lugar como una alternativa sustentable para la generación de energía eléctrica.

\section{ANTECEDENTES}

Experiencia desarrollada por el CER-UNI en actividades tecnológicas orientadas a contribuir con el mejoramiento de la calidad de vida de pobladores en comunidades rurales y alejadas del Perú.

Desde el año 2004, la Universidad Nacional de Ingeniería (UNI), a través del Centro de Energías Renovables de la UNI (CER-UNI), ha llevado a cabo proyectos en la comunidad rural San Francisco de Raymina utilizando las energías renovables como fuente de energía para la aplicación de diversas tecnologías limpias y renovables.

Los proyectos estuvieron enfocados en promover el desarrollo de actividades productivas, pero también en mejorar las condiciones de confort térmico de las viviendas para hacer frente a las condiciones climáticas extremas de baja temperatura de la zona.

\subsection{DESCRIPCIÓN GENERAL DE LA COMUNIDAD}

Se ubica en el distrito de Huambalpa, Provincia de Vilcashuamán - Región Ayacucho, a 3798 msnm., con coordenadas: latitud $13^{\circ} 44^{\prime} 34.70^{\prime \prime}$ Sur y longitud $73^{\circ} 53^{\prime} 04.50^{\prime \prime}$ Oeste, con los siguientes parámetros meteorológicos promedios: temperatura $9.3{ }^{\circ} \mathrm{C}$, humedad relativa $69.2 \%$, irradiación solar diaria de 249.2 W. $\mathrm{m}^{-2}$ (promediado sobre 24 horas) [10]. La temporada de lluvias ocurre entre los meses de diciembre y abril, donde ocasionalmente se producen tormentas eléctricas y granizadas. El resto del año es seco. La localidad está conformada por 35 familias con una población total de 109 habitantes ( $52.3 \%$ son mujeres), las viviendas son de paredes de adobe y techos de calamina (aproximadamente: $350 \mathrm{~m}^{2}$ cada vivienda); tiene una escuela primaria (con 18 niños, de edades comprendidas entre 5 a 11 años), y un local comunal, construida con material noble [11].

En lo referente al nivel educativo de la población, solo el $31.8 \%$ de los pobladores terminaron la escuela secundaria. La mayoría de la población se dedica a la agricultura y en sus campos siembran tubérculos, hortalizas y cereales; toda la producción es para autoconsumo. La población pecuaria en la comunidad está constituida principalmente por vacunos, ovinos, porcinos y aves de corral. Los ingresos, en términos económicos, provienen por la venta de ganado vacuno u ovino. Además, la comunidad recibe el apoyo de los programas sociales que el Estado peruano promueve en las zonas de pobreza. La comunidad dispone de los servicios básicos de energía eléctrica y agua potable, pero carece de desagüe; las necesidades fisiológicas se realizan en letrinas de silo seco [12]. La población no cuenta con una posta médica. Las vías de comunicación terrestre con otras poblaciones son a través de 
carreteras afirmadas. La comunidad cuenta con algunas tecnologías para actividades productivas, implementadas con energías renovables, pero por diversos motivos, mayormente se encuentran inoperativas.

La comunidad San Francisco de Raymina (Fig. 1) tiene el acceso al servicio de energía eléctrica desde el año 2006; la energía proviene del Sistema Eléctrico (SE0068) Cangallo-Llusita, localizada a una distancia aproximada de $70 \mathrm{~km}$ y es suministrado por la empresa Electrocentro S.A.

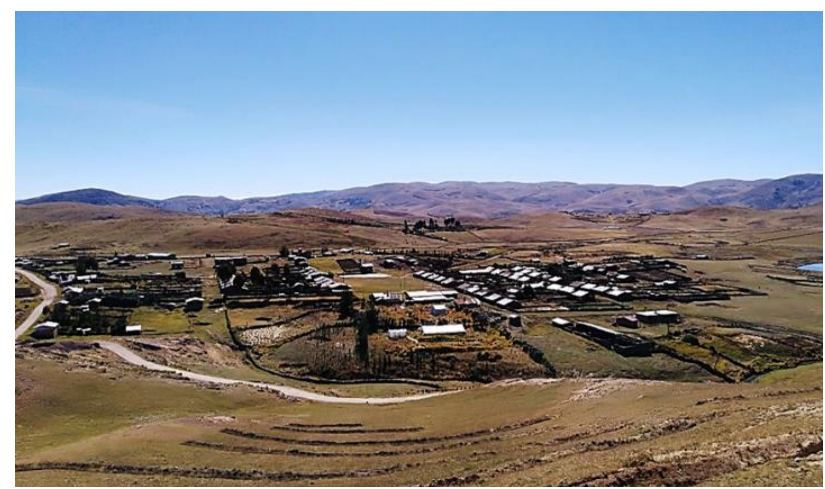

Fig. 1. Vista panorámica de la comunidad San Francisco de Raymina.

La infraestructura y la tarifa eléctrica en la comunidad se encuentran subsidiadas a través del Fondo de Compensación Social Eléctrica (FOSE).

La tabla I muestra a modo de referencia los costos de inversión para la electrificación de viviendas de la localidad (35 familias), basada en proyectos de electrificación rural desarrollados en la Región Ayacucho por la DGER/MEM.

TABLA I

Inversión estimada para electrificación de viviendas en zonas rurales de Ayacucho.

\begin{tabular}{ccccc}
\hline $\begin{array}{c}\mathrm{N}^{\circ} \text { de } \\
\text { usuarios }\end{array}$ & $\begin{array}{c}\text { Línea } \\
\text { primaria } \\
\text { USD }\end{array}$ & $\begin{array}{c}\text { Redes } \\
\text { primarias } \\
\text { USD }\end{array}$ & $\begin{array}{c}\text { Redes } \\
\text { secundarias } \\
\text { USD }\end{array}$ & $\begin{array}{c}\text { Costo de } \\
\text { Inversión } \\
\text { USD }\end{array}$ \\
\hline 35 & 6507.00 & 7310.00 & 11443.00 & 25260.00 \\
\hline
\end{tabular}

(Elaborada sobre la base de la información de Electrocentro S.A.)

La comunidad cuenta con energía eléctrica muy limitada, por una línea de $13.2 \mathrm{kV}, 60 \mathrm{~Hz}$, monofásico con retorno por tierra (MRT), que llega a un transformador de 5 kVA localizado en la comunidad (Fig. 2). Del transformador se distribuye con una red local, conexión aérea de baja tensión (BT), de $220 \mathrm{~V}, 60 \mathrm{~Hz}$, monofásico para 35 viviendas y 5 postes de alumbrado público de $50 \mathrm{~W} \mathrm{c/u}$.

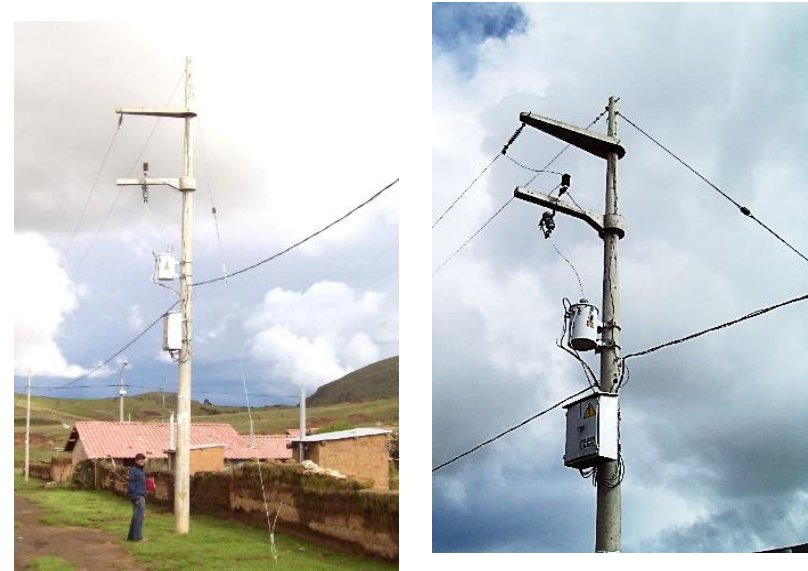

Fig. 2. Vista del transformador de distribución monofásico de 5 kVA localizado en la comunidad de Raymina.

\section{METODOLOGÍA}

La metodología empleada en este trabajo se basó en el análisis de tres puntos, aplicados a la comunidad rural altoandina San Francisco de Raymina en Ayacucho:

\section{a) Diagnóstico del servicio eléctrico}

Se determinó y analizó el uso y costo de energía, basado en encuestas domiciliarias, dirigidas al jefe del hogar para identificar las necesidades eléctricas de cada familia y de la población en general. Se analizó la cobertura actual del servicio y se detalla la configuración de la red eléctrica.

\section{b) Evaluación del recurso energético solar}

Se estudió el potencial energético solar aprovechable de la localidad. Para la evaluación del recurso se analiza los registros de datos de una estación meteorológica (HOBO Micro Station Data Logger H21-002), instalada en la comunidad por el CER-UNI. Con la información obtenida se realiza un procesamiento estadístico de datos y posteriormente se estima el potencial de energía solar del lugar.

\section{c) Diseño de una planta fotovoltaica}

Finalmente, sobre la base de los resultados del diagnóstico del servicio eléctrico y de la información sobre el potencial energético solar aprovechable del lugar, se elaboró una propuesta tecnológica para cubrir la demanda de energía eléctrica de toda la comunidad, la cual consiste en el diseño de un sistema fotovoltaico de $4.5 \mathrm{~kW}_{\mathrm{p}}$, conectado a la red local y con capacidad de almacenamiento.

\section{ANÁLISIS DE RESULTADOS}

\subsection{DEMANDA DE ENERGÍA EN LA COMUNIDAD}


C. Salvador et al.

El $95.8 \%$ de las familias cuentan con energía eléctrica. $36 \%$ del consumo de energía eléctrica en los hogares es para iluminación y $64 \%$ para electrodomésticos (TV color, DVD, radio, licuadora, plancha, equipo de sonido y cargador de celular). El costo de consumo de energía en la comunidad resulta ser S/ 0.426 por kWh. La curva potencia vs tiempo (Fig. 3) muestra el perfil típico de consumo eléctrico diario de toda la comunidad.

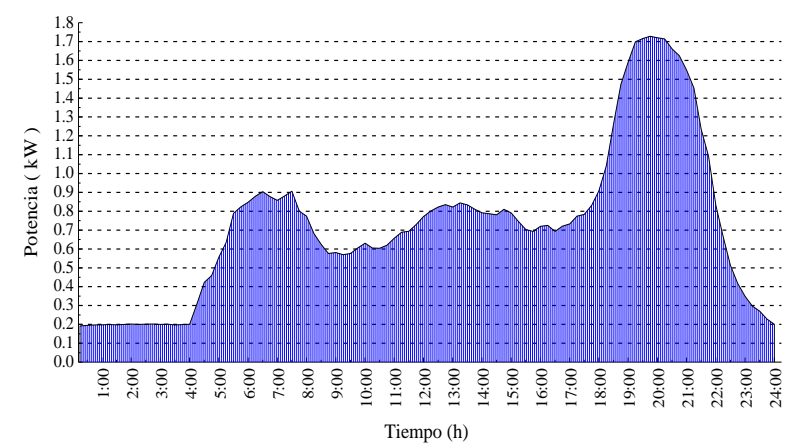

Fig. 3. Perfil típico diario de la potencia del consumo de electricidad de la comunidad San Francisco de Raymina (Elaborado sobre la base de la información brindada por la empresa Electrocentro S.A.).

El promedio diario del consumo de energía eléctrica de toda la comunidad, incluyendo el alumbrado público, resulta ser $17.56 \mathrm{kWh}$, esta se calcula integrando la curva potencia vs tiempo del perfil típico diario de consumo de electricidad. El promedio mensual del consumo de energía eléctrica por familia es $12.50 \mathrm{kWh}$. El servicio tiene en promedio un costo por familia de S/ 10.0 por mes.

\subsection{EVALUACIÓN DEL RECURSO ENERGÉTICO SOLAR}

Se empleó una estación meteorológica (HOBO Micro Station Data Logger H21-002) para el registro de datos climatológicos del lugar [13]. De acuerdo con los resultados de la evaluación del recurso energético solar, los índices de radiación solar diaria media mensual varían entre $4.1 \mathrm{kWh} \cdot \mathrm{m}^{-2} \cdot \mathrm{día}^{-1}$ y $6.2 \mathrm{kWh} \cdot \mathrm{m}^{-2} \cdot \mathrm{día}^{-1}$, los cuales se encuentran registrados en los meses de mayo y octubre, respectivamente. La tabla II muestra la radiación solar acumulada por mes que se registra en la comunidad. La radiación solar diaria media anual que recibe la comunidad resulta ser $5.3 \mathrm{kWh} \cdot \mathrm{m}^{-2} \cdot \mathrm{día}^{-1}$ a una altitud de 3798 msnm.

TABLA II

Energía Solar diaria promedio mensual en la localidad.

\begin{tabular}{lc}
\hline Mes & $\begin{array}{c}\text { Energía solar } \\
\mathrm{kWh} \cdot \mathrm{m}^{-2} \cdot \mathrm{dí}^{-1}\end{array}$ \\
\hline Enero & 5.21 \\
Febrero & 4.10 \\
Marzo & 5.06
\end{tabular}

DOI: https://doi.org/ 10.21754/tecnia.v2111.1104

\begin{tabular}{ll} 
Abril & 5.46 \\
Mayo & 4.56 \\
Junio & 5.55 \\
Julio & 6.07 \\
Agosto & 5.50 \\
Setiembre & 5.48 \\
Octubre & 6.22 \\
Noviembre & 6.06 \\
Diciembre & 4.28 \\
\hline Promedio anual & 5.30 \\
\hline
\end{tabular}

Para asegurar la confiabilidad de la información obtenida, se ha comparado con los datos de la radiación solar diaria media anual disponible en The POWER Project de la NASA [14], los que indican para Raymina una radiación solar diaria media anual de $5.32 \mathrm{kWh} \cdot \mathrm{m}^{-}$ ${ }^{2} \cdot$ día $^{-1}$,

a una altitud de $3525.6 \mathrm{msnm}$.

\subsection{PROPUESTA DE UNA PLANTA FOTOVOLTAICA PARA SAN FRANCISCO DE RAYMINA}

Se propone la implementación de un sistema fotovoltaico de $4.5 \mathrm{~kW}$, conectado a la red local y con capacidad de almacenamiento, como una alternativa sustentable para complementar la red convencional actual, que presenta muchas interrupciones. De acuerdo con los resultados del diagnóstico energético, la oferta de energía eléctrica para toda la comunidad resulta ser actualmente $17.56 \mathrm{kWh} \cdot \mathrm{día}^{-1}$. En la tabla III se presenta el presupuesto económico para un sistema fotovoltaico que produce esta energía eléctrica; es decir, esta planta solar cubrirá la energía eléctrica actualmente disponible en la comunidad. Se propone su instalación inicialmente como complemento del sistema existente (conexión a la red nacional), garantizando así $17.56 \mathrm{kWh} \cdot \mathrm{día}^{-1}$ y duplicando la disponibilidad de energía eléctrica si funciona la conexión a la red nacional, con miras a ampliarla en el futuro y eliminar el sistema costoso de la red convencional actual.

\section{a) Características técnicas de la planta fotovoltaica}

(Fig. 4) describe la planta fotovoltaica de $4.5 \mathrm{~kW}$, conectado a la red local y con capacidad de almacenamiento, propuesta para la comunidad San Francisco de Raymina.

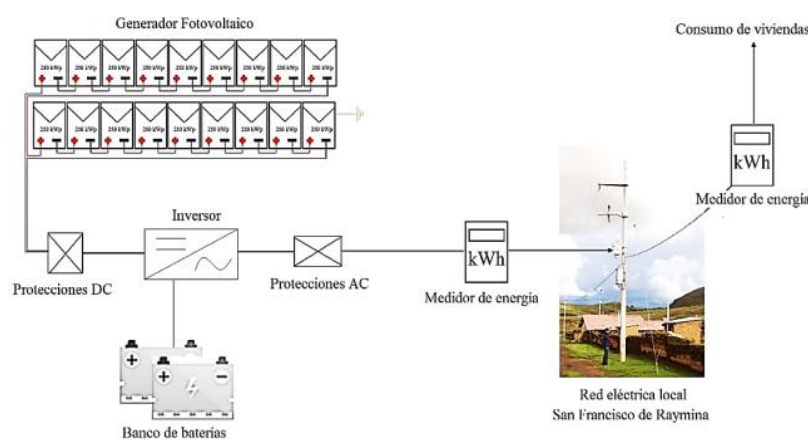

Journal TECNIA Vol.31 N² January-June 2021 
C. Salvador et al.

Fig. 4. Esquema de instalación de la planta fotovoltaica.

- Potencia del generador fotovoltaico (PGFv). Se calcula con base en la información del consumo promedio de energía eléctrica diario de toda la comunidad de Raymina (17.56 kWh.día-1) y de la radiación solar diaria media anual que recibe la localidad ( $\left.5.30 \mathrm{kWh} \cdot \mathrm{m}^{-2} \cdot \mathrm{día}^{-1}\right)$. Para ello, se eligieron módulos policristalinos de la marca SolarWorld modelo SW 250 (resultan más accesibles en el mercado local) y se emplearán un total de 18 módulos fotovoltaicos para obtener una potencia nominal de $4.5 \mathrm{~kW}_{\mathrm{p}}$.

- Potencia del inversor. Basado en la potencia del generador fotovoltaico, el inversor solar elegido para la planta fotovoltaica es de la marca SMA Sunny Mini Central 5000A, que tiene una potencia máxima de salida $A C$ de $5 \mathrm{~kW}$. La potencia de entrada del inversor ( $P_{I N V}, D C \approx P_{I N V}, A C$, en $W$ ) depende de la potencia en Condiciones Estándar de Medida (CEM) del GFV mediante el factor de dimensionado (Fs). Usualmente, $0.7<\mathrm{Fs}_{\mathrm{s}}<1.2$ [15]. La elección de un valor específico de $F_{s}$ no altera significativamente la producción de electricidad AC. Como la planta fotovoltaica va ser implementado en una zona de clima muy frio a una altura de $3800 \mathrm{msnm}$., para evitar problemas y deficiencias en el correcto funcionamiento de nuestra instalación, es recomendable hacer un sobredimensionamiento de la potencia del inversor que contemple los picos de arranque, por tal motivo el Fs debe ser mayor a 1.

- Banco de baterías. Las baterías más eficientes en la actualidad son las de tecnología de Litio. Para la planta fotovoltaica se ha elegido la batería solar de litio LG Chem RESU 48V 9.8 kWh, con una energía utilizable de 8.8 kWh. Se emplearán 2 baterías de Litio para que el sistema tenga una capacidad de almacenamiento de $17.6 \mathrm{kWh}$. Los acumuladores de energía sirven de respaldo en horarios o momentos donde no haya disponibilidad solar (por las noches y días nublados).

- Inclinación óptima de los módulos ( $\beta$ ). La inclinación óptima que deben tener los módulos fotovoltaicos en función de la latitud del lugar $(\varphi)$, se calcular de la siguiente manera:

$$
\beta=0.69 \cdot|\varphi|+3.7^{\circ}
$$

Reemplazando datos en la ecuación (1):

$$
\beta=13.2^{\circ}
$$

- Separación mínima entre 2 filas de módulos. El generador fotovoltaico se encuentra conformado por 18 módulos de $250 \mathrm{~W}_{\mathrm{p}} \mathrm{c} / \mathrm{u}$, distribuidos en
2 cadenas por serie conectadas en paralelo (9 módulos por serie-cadena). La distancia mínima entre las 2 dos filas de módulos resulta ser $2.0 \mathrm{~m}$. Los módulos se ubicaran sobre soportes metálicos fijos.

- Producción de energía eléctrica estimada $\left(E_{A C}\right)$. Se determina empleando la siguiente ecuación:

$E_{A C}=P_{G F V, S T C} \cdot$

Donde:

$E_{A C}$ : Energía eléctrica generada anualmente (kWh).

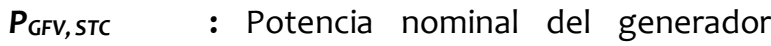
fotovoltaico en condiciones estándar en el punto de máxima potencia $\left(\mathrm{kW}_{\mathrm{p}}\right)$.

$\boldsymbol{H}_{d a}(\boldsymbol{\alpha}, \boldsymbol{\beta})$ : Valor medio anual de la irradiación diaria sobre la superficie del generador fotovoltaico $\left(\mathrm{Wh} \cdot \mathrm{m}^{-2}\right)$.

Gstc: Irradiancia en condiciones estándar $\left(1000 \mathrm{~W} \cdot \mathrm{m}^{-2}\right)$.

PR: Factor de rendimiento anual (0.75).

Reemplazando datos en la ecuación (2):

$$
E_{A C}=6528.94 k W h
$$

- Productividad final anual $\left(\mathrm{Y}_{\mathrm{F}}\right)$. Se determina de la siguiente manera:

$Y_{F}=\frac{E_{A C}(k W h)}{P_{G F V}\left(k W_{P}\right)}$

Reemplazando datos en la ecuación (3):

$$
Y_{F}=1450.88 \mathrm{kWh} / \mathrm{k} W_{P}
$$

- Productividad de referencia anual $\left(Y_{R}\right)$. Se expresa en $\left(\mathrm{kWh} / \mathrm{m}^{2}\right) /\left(\mathrm{kW} / \mathrm{m}^{2}\right)$, se calcula de la siguiente forma:

$Y_{R}=H_{d a}(\alpha, \beta) / G_{S T C}$

Reemplazando datos en la ecuación (4):

$$
Y_{R}=1934.50\left(k W h / m^{2}\right) /\left(k W / m^{2}\right)
$$

- Coeficiente global de rendimiento anual (PR). Es un indicador de calidad. Se define como la relación entre la productividad final y la productividad de referencia. Se determina de la siguiente manera:

$$
P R=\frac{Y_{F}}{Y_{R}}
$$

Reemplazando datos en la ecuación (5):

$$
P R=\frac{1450.88 k W h / k W_{P}}{1934.50\left(k W h / m^{2}\right) /\left(k W / m^{2}\right)}=0.75
$$


C. Salvador et al.

\section{b) Costo de la planta fotovoltaica}

La tabla III presenta el presupuesto económico para la implementación de la planta fotovoltaica. Esta propuesta tecnológica, descrita anteriormente, cubriría la demanda de energía eléctrica total de la localidad.

TABLA III

Presupuesto económico para la implementación de una planta fotovoltaica de $4.5 \mathrm{~kW}_{\mathrm{p}}$.

\begin{tabular}{|c|c|c|c|}
\hline Item & Cant. & $\begin{array}{l}\text { Precio } \\
\text { Unit. } \\
\text { USD }\end{array}$ & $\begin{array}{l}\text { Importe } \\
\text { USD }\end{array}$ \\
\hline Panel Fotovoltaico de $250 \mathrm{~W}_{\mathrm{p}}$ & 18 & 210 & 3780 \\
\hline Batería de Litio 9.8 kWh & 2 & 7105 & 14210 \\
\hline Inversor mini central $5 \mathrm{~kW}$ & 1 & 2990 & 2990 \\
\hline Medidor de energía & 2 & 120 & 240 \\
\hline Protecciones eléctricas, cables & 1 & 390 & 390 \\
\hline Sistema puesta a tierra & 1 & 410 & 410 \\
\hline Estructura de soporte fija & 1 & 1200 & 1200 \\
\hline $\begin{array}{l}\text { Mano de obra, transporte de } \\
\text { materiales }\end{array}$ & 1 & 790 & 790 \\
\hline Tipo de cambio: USD $1.0=$ S/ 3.50 & & Total & 24010.00 \\
\hline
\end{tabular}

- Mantenimiento de la planta fotovoltaica. El mantenimiento para la planta fotovoltaica es de carácter preventivo y correctivo. Consiste en la limpieza periódica de los componentes para garantizar su buen funcionamiento. No requiere cambios de componentes. La frecuencia prevista es trimestral y tiene un costo estimado de USD 48,o por año (incluye: personal + materiales de limpieza).

- Costo energético nivelado (LCOE). Es el valor neto actual del costo unitario de la electricidad durante la vida útil de la planta fotovoltaica. Se requiere conocer dos variables:

Costo total del sistema (financiamiento + mantenimiento).

\section{¿Cuánta energía producirá la planta fotovoltaica durante el período útil?}

Su cálculo viene dado por:

$$
L C O E=\frac{(\text { Costofijoanual }+ \text { costosvariablesanual })}{\text { Generacióntotaldeenergía }}\left(\frac{U S D}{k W h}\right)
$$

Para la planta fotovoltaica se cuenta con la siguiente información técnica:

Costo total de la planta fotovoltaica: O\&M por 30 años:

USD 24010

Producción de energía eléctrica

(30 años):

USD 1440

$195868.2 \mathrm{kWh}$

Reemplazando datos en la ecuación (6):

$$
\begin{gathered}
L C O E=\frac{(24010+1440)}{195868,2}=\frac{25450}{195868.2}\left(\frac{U S D}{k W h}\right) \\
L C O E=0.130\left(\frac{U S D}{k W h}\right)
\end{gathered}
$$

La tabla IV resume las características principales de la planta fotovoltaica propuesta para Raymina.

\begin{tabular}{|c|c|c|}
\hline Característica & Valor & Unidad \\
\hline Potencia del generador fotovoltaico & 4.5 & $\mathrm{~kW}_{\mathrm{p}}$ \\
\hline Potencia del inversor & 5.0 & $\mathrm{~kW}$ \\
\hline Banco de baterías & 19.6 & $\mathrm{kWh}$ \\
\hline Producción anual de energía estimado & 6528.94 & $\mathrm{kWh}$ \\
\hline Productividad final anual $\left(\mathrm{Y}_{\mathrm{F}}\right)$ & 1450.88 & $\mathrm{kWh} / \mathrm{kW}_{\mathrm{p}}$ \\
\hline Productividad de referencia anual $\left(Y_{R}\right)$ & 1934.50 & horas \\
\hline $\begin{array}{l}\text { Coeficiente global de rendimiento anual } \\
\text { (PR) }\end{array}$ & 0.75 & \\
\hline Costo energético nivelado & 0.130 & USD/kWh \\
\hline $\begin{array}{l}\text { Tiempo de vida útil estimada de la } \\
\text { planta fotovoltaica }\end{array}$ & 30 & años \\
\hline
\end{tabular}

TABLA IV

Principales características de la planta fotovoltaica.

A partir de los resultados, se presenta una comparativa de costos de inversión entre 2 sistemas para suministrar energía eléctrica a la localidad (35 familias). La tabla $\vee$ muestra los costos de inversión.

TABLA V

Inversión estimada para la electrificación de viviendas de la localidad.

\begin{tabular}{lcc}
\hline Sistema de suministro de energía & $\begin{array}{c}\text { Costo de } \\
\text { inversión } \\
\text { USD }\end{array}$ & $\begin{array}{c}\text { Costo } \\
\text { (O\&M)/anual } \\
\text { USD }\end{array}$ \\
\hline $\begin{array}{l}\text { Red convencional actual } \\
\text { Planta fotovoltaica de } 4.5 \mathrm{~kW}_{\mathrm{p}},\end{array}$ & 260.00 & 1010.40 \\
$\begin{array}{l}\text { conectado a la red local y con } \\
\text { capacidad de almacenamiento }\end{array}$ & 24010.00 & 48.00 \\
\hline
\end{tabular}

\section{CONCLUSIONES}

- Se identificaron las necesidades de energía eléctrica de la comunidad San Francisco de Raymina: la localidad no cuenta con suficiente energía eléctrica para el desarrollo de actividades productivas. Asimismo, en promedio las familias consumen 12.5 kWh por mes y toda la comunidad consume $6409.40 \mathrm{kWh} /$ anual.

- El consumo de electricidad es exclusivamente para iluminación y el uso de electrodomésticos. Los pobladores anhelan contar con mayor energía en sus viviendas para el uso de equipos de mayor potencia.

- La localidad presenta un alto potencial de energía solar, el cual puede ser aprovechado para la aplicación de tecnologías fotovoltaicas y cubrir la demanda insatisfecha de energía eléctrica de manera sustentable.

- La producción de energía eléctrica estimado por la planta fotovoltaica es de $6528.94 \mathrm{kWh}$ por año. 
C. Salvador et al.

- La planta fotovoltaica propuesta es una alternativa tecnológica limpia y viable, que tiene por finalidad dar solución al problema de déficit de energía eléctrica que presenta la localidad, a fin de contribuir y promover el desarrollo de actividades productivas de manera sustentable.

\section{AGRADECIMIENTOS}

Para el desarrollo de este trabajo, se contó con la colaboración de los dirigentes comunales y población en general de la comunidad rural altoandina San Francisco de Raymina. Además, se tuvo la colaboración del CER-UNI, nos facilitó los registros de datos de la estación meteorológica (HOBO Micro Station Data Logger H21-002), instalada en la localidad.

\section{REFERENCIAS}

[1] INEI (2019). Informe Técnico: Evolución de la pobreza monetaria 2007-2018 [Online]. Available: https://www.inei.gob.pe/media/MenuRecursivo/publicaciones_digital es/Est/Lib1646/libro.pdf.

[2] R. Escobar, D. Vilar, E. Velo, L. Ferrer M., y B. Domenech, "Promoting and Improving Renewable Energy Projects Through Local Capacity Development", in Modelling and Optimization of Renewable Energy Systems; In Tech: Rijeka, Croatia, 2012; pp. 146-147.

[3] M. Schäfer, N. Kebir y K. Neumann, "Research needs for meeting the challenge of decentralized energy supply in developing countries", Energy Sustain. Dev., vol. 15, no. 3, pp. 324-329, sept. 2011.

[4] S. Belmonte, J. Franco, J. Viramonte, y V. Núñez, "Integración de las energías renovables en procesos de ordenamiento territorial", Av. energ. renov. medio ambiente, vol. 13, pp. 07-41, 2009.

[5] R. Ladino, "La energía solar fotovoltaica como factor de desarrollo en zonas rurales de Colombia. Caso: Vereda Carupana, municipio de Tauramena, departamento de Casanare", Tesis de maestría, Pontificia Universidad Javeriana, Bogotá-Colombia, 2011.

[6] R. Caminada, "Energías renovables: el desarrollo de la energía eólica en el Perú", Universidad de San Martin de Porres, Facultad de Derecho, 2016.

[7] R. E. Mitma, "Análisis de la Regulación de Energías Renovables en el Perú", Derecho Soc., no. 45, pp. 167-176, 2015.

[8] M. Horn (2009). Aprovechamiento descentralizado de fuentes renovables de energía [Online]. Available: http://fc.uni.edu.pe/mhorn/Aprovechamiento\%20descentralizado.pdf. [9] R. Escobar et al., "Energización rural mediante el uso de energías renovables para fomentar un desarrollo integral y sostenible: Propuestas para alcanzar el acceso universal a la energía en el Perú", Coop. Alem. al Desarro. - Agencia la GIZ en el Perú, vol. primera ed, 2016. [10] J. O. Molina, R. E. Espinoza, M. J. Horn, y M. M. Gómez, "Thermal performance evaluation of isolation and two active solar heating systems for an experimental module: A rural Peruvian case at 3700 masl", J. Phys.: Conf. Ser., vol. 1173, p. 012003, feb. 2019.

[11] R. Espinoza, J. Molina, M. Horn, y M. M. Gómez, "Conceptos bioclimáticos y su aplicabilidad a la zona rural altoandina: Caso comunidad San Francisco de Raymina (SFR)-Ayacucho", tecnia, vol.25, no.2, p.5, dic. 2015.

[12] R. Espinoza, "Evaluación experimental de la performance de dos componentes bioclimáticas de calentamiento aplicadas en un módulo de vivienda de San Francisco de Raymina - Ayacucho con el propósito de validarlas como técnicas de estrategias bioclimáticas para viviendas rurales Alto Andinas", Tesis de maestría, Universidad Nacional de Ingeniería, Lima-Perú, 2014.

[13] Onset (2019). HOBO Micro Station Data Logger [Online]. Available: https://www.onsetcomp.com/products/data-loggers/h21$\underline{002 .}$
[14] NASA (2021, enero 23). POWER Data Access Viewer [Online]. Available: https://power.larc.nasa.gov/data-access-viewer/.

[15] APES (2014). Dimensionado de un SFCR (I) Generador fotovoltaico - Inversor, Cursos XXI SPES - PIURA 2014 [Online]. Available: http://www.perusolar.org/cursos-xxi-spes-piura-2014/.
Los artículos publicados por TECNIA pueden ser compartidos a través de la licencia Creative Commons: CC BY 4.o. Permisos lejos de este alcance pueden ser consultados a través del correorevistas@uni.edu.pe 